\title{
Anatomical Correlates of Directional Hypokinesia in Patients with Hemispatial Neglect
}

\author{
Ayelet Sapir, ${ }^{1}$ Julie B. Kaplan, ${ }^{4}$ Biyu J. He, ${ }^{1}$ and Maurizio Corbetta ${ }^{1,2,3}$ \\ Departments of ${ }^{1}$ Neurology, ${ }^{2}$ Radiology, and ${ }^{3}$ Anatomy and Neurobiology, Washington University School of Medicine, St. Louis, Missouri 63110, and \\ ${ }^{4}$ Department of Psychology, Washington University, St. Louis, Missouri 63110
}

\begin{abstract}
Unilateral spatial neglect (neglect) is a syndrome characterized by perceptual deficits that prevent patients from attending and responding to the side of space and of the body opposite a damaged hemisphere (contralesional side). Neglect also involves motor deficits: patients may be slower to initiate a motor response to targets appearing in the left hemispace, even when using their unaffected arm (directional hypokinesia). Although this impairment is well known, its anatomical correlate has not been established. We tested 52 patients with neglect after right hemisphere stroke, and conducted an anatomical analysis on 29 of them to find the anatomical correlate of directional hypokinesia. We found that patients with directional hypokinesia had a lesion involving the ventral lateral putamen, the claustrum, and the white matter underneath the frontal lobe. Most importantly, none of the patients without directional hypokinesia had a lesion in the same region. The localization of neglect's motor deficits to the basal ganglia establishes interesting homologies with animal data; it also suggests that a relative depletion of dopamine in the nigrostriatal pathway on the same side of the lesion may be an important pathophysiological mechanism potentially amenable to intervention.
\end{abstract}

Key words: anatomy; attention; basal ganglia; fMRI; motor; neglect; stroke

\section{Introduction}

Neglect is a clinical syndrome characterized by an inability to attend and respond to objects on the side of space opposite to a lesioned hemisphere (contralesional) (Heilman et al., 1993). It appears in 25\% of stroke patients (Pedersen et al., 1997) and is more common after a right hemisphere lesion, with an incidence of $\sim 50 \%$ (Buxbaum et al., 2004), than after a left hemisphere lesion. The affected patients might fail to eat from the left side of the tray, have trouble grooming the left side of their body, and respond poorly to people standing on their left. These deficits are thought to be related to a perceptual/attentional deficit toward stimuli in the left visual field (VF). The most common lesion sites are the right inferior parietal lobule and ventral frontal and superior temporal gyrus (Heilman et al., 1983; Vallar and Perani, 1986; Husain and Kennard, 1996; Karnath et al., 2001; Mort et al., 2003); subcortical regions such as the thalamus and the basal ganglia also cause neglect (Karnath et al., 2002, 2004).

In addition to attention-perception deficits, patients with neglect may show slowed initiation of the ipsilesional arm toward the contralesional space, compared with movements directed toward the ipsilesional space [directional hypokinesia (DH)]. Although this may reflect perceptual deficits, it has been suggested that $\mathrm{DH}$ underlies a true motor planning deficit unrelated to

Received July 12, 2006; revised Feb. 15, 2007; accepted Feb. 19, 2007.

This work was supported by the J. S. McDonnell Foundation, the J. S. McDonnell Center for Higher Brain Foundation, and the National Institute of Neurological Disorders and Stroke. We thank Giovanni d'Avossa for assistance with statistical issues.

Correspondence should be addressed to Ayelet Sapir, Department of Neurology, Washington University School of Medicine, 4525 Scott Avenue, St. Louis, M0 63110. E-mail: ayelet@npg.wustl.edu. DOI:10.1523/JNEUROSCI.0041-07.2007

Copyright $\odot 2007$ Society for Neuroscience $\quad$ 0270-6474/07/274045-07\$15.00/0 perception (Heilman et al., 1985). Standard tests of neglect, such as cancellation tasks and line bisection, are unable to distinguish between perceptual and motor components, because they require a motor response to be made in the neglected hemifield. Many studies have tried to solve this problem using a variety of ingenious solutions (Heilman et al., 1985; Bisiach et al., 1990; Coslett et al., 1990; Mijoviâc, 1991; Tegner and Levander, 1991; Chiba et al., 2005). Most studies found that patients with a frontal lobe lesion exhibit DH (Daffner et al., 1990; Tegner and Levander, 1991; Bottini et al., 1992) (but see Ishiai et al., 1994) (for review, see Vallar, 2001).

Mattingley et al. (1998) challenged this observation by introducing a new method for separating perceptual and motor components of neglect. In their reaching task, targets could appear either on the right or the left of a fixation point and patients had to reach with their right hand toward the target. The hand starting position was manipulated in separate blocks. When subjects reach to left visual field targets from a midline position, they use a leftward trajectory. In this condition, perceptual and motor neglect are coincident. However, when the starting hand position is to the left of the left target, a left target would still fall in the contralesional visual field, but now subjects perform a rightward reach to localize it. Therefore, in this case, perceptual (i.e., stimulus analysis) and motor (i.e., preparation of a reach) are dissociated. Mattingley et al. (1998) tested three patients with inferior parietal and three with inferior frontal lesion and found that parietal patients showed $\mathrm{DH}$, whereas patients with frontal lesion showed only perceptual neglect. In the current study, we examined the anatomoclinical correlates of $\mathrm{DH}$ in a larger sample of neglect patients using a variant of this reaching task. 


\section{Materials and Methods}

Patients

Fifty-two stroke patients (29 men) with cortical and subcortical lesions who showed neglect in a screening procedure participated in the study. Mean \pm SD age was $59.3 \pm 15.03$ years. Inclusion and exclusion criteria for patients are listed below. Thirty age-matched controls (14 men) without neurological or psychiatric conditions were also tested. Mean \pm SD age was $64.2 \pm 15.04$ years.

Inclusion criteria. (1) Age 18 or greater. No upper age limit was applied. (2) Single right hemisphere lesion, ischemic or hemorrhagic in etiology. (3) Clinical evidence of neglect during clinical screening. The clinical screening included evidence of extinction in visual, auditory, or tactile domains in a neurological exam, evidence of neglect in activities of daily living as reported by the treating occupational therapist at the time of enrollment, and a failure to cancel targets among distractors in a behavioral inattention test (BIT) star cancellation task (see below). (4) Time of enrollment: $>1$ week and $<4$ weeks from stroke onset. (5) Awake, alert, and capable of understanding and participating in research.

Exclusion criteria. (1) Evidence by computer tomography or magnetic resonance imaging (MRI) of other strokes, although up to two lacunes were allowed in the white matter. (2) Inability to maintain wakefulness. (3) Presence of other neurological, psychiatric, or medical conditions that would preclude active participation in research and/or alter the interpretation of the behavioral/imaging studies (e.g., dementia; see below) or limit life expectancy to $<1$ year (e.g., cancer or congestive heart failure class IV). (4) Report of claustrophobia excluded patients from the MRI study and therefore from the lesion anatomy analysis. Some patients did not come back for anatomy scans. From the present analysis of lesion anatomy, we also excluded one patient with a visual cortex lesion. This led to a total of 29 patients for whom we had behavioral and anatomical data.

\section{Procedure}

Before conducting the main experiment, all patients performed the following neuropsychological tests.

The Short Blessed scale (Katzman et al., 1983) is a test of orientation, memory, and concentration. We used this task to rule out patients with dementia (score $<8$ ). Bisiach personal neglect assessment is a proprioceptive identification task in which the patient is asked to locate one hand with the other (Bisiach et al., 1986). The fluff task is a visuospatial test of personal neglect (Cocchini et al., 2001). The patient is blindfolded, and six cotton balls are placed on the contralesional side of the patient's body. The blindfold is removed, and the patient is asked to find the cotton balls. The number of cotton balls found by the patient is counted. BIT stars and Mesulam shape cancellation are both tests of visual spatial scanning designed to evaluate hemispatial neglect. BIT star (Wilson et al., 1987) consists of an array of small and large stars. The patient is instructed to circle all of the small stars. The patient is scored according to the number of small stars omitted in the left visual field minus the number omitted in the right visual field as well as the total numbers of omissions. Mesulam shape cancellation (Mesulam, 1985) is similar but with multiple shapes and distractors. The patient is asked to circle all of the targets of one particular shape. In BIT reading (Wilson et al., 1987), an article containing three columns (left, center, and right) is presented at body midline. The patient is instructed to read the article out loud. The number of omitted words in each column is counted. In clock drawing (Wilson et al., 1987), patients are asked to draw a clock with all of the numbers on a blank paper and to set the time to a specific hour. The number of mistakes is measured. In the baking tray (Tham and Tegner, 1996) task, patients were asked to place 16 plastic cookies on a tray as symmetrically as possible. Points were given for each cookie in the correct place.

In addition, all patients performed computerized tasks for testing vigilance and spatial orienting.

\section{Perceptual neglect and directional hypokinesia}

Apparatus and stimuli. The subjects sat in a dim room facing a computer screen $60 \mathrm{~cm}$ in front of them at eye level. The subject's index finger of the preferred hand rested on a response key placed on the table between the subject and the screen. A constant display consisted of a fixa-
A.
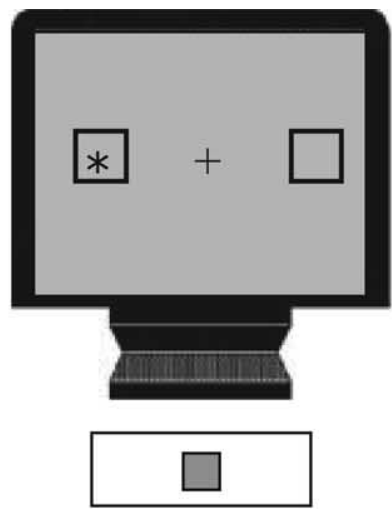

B.
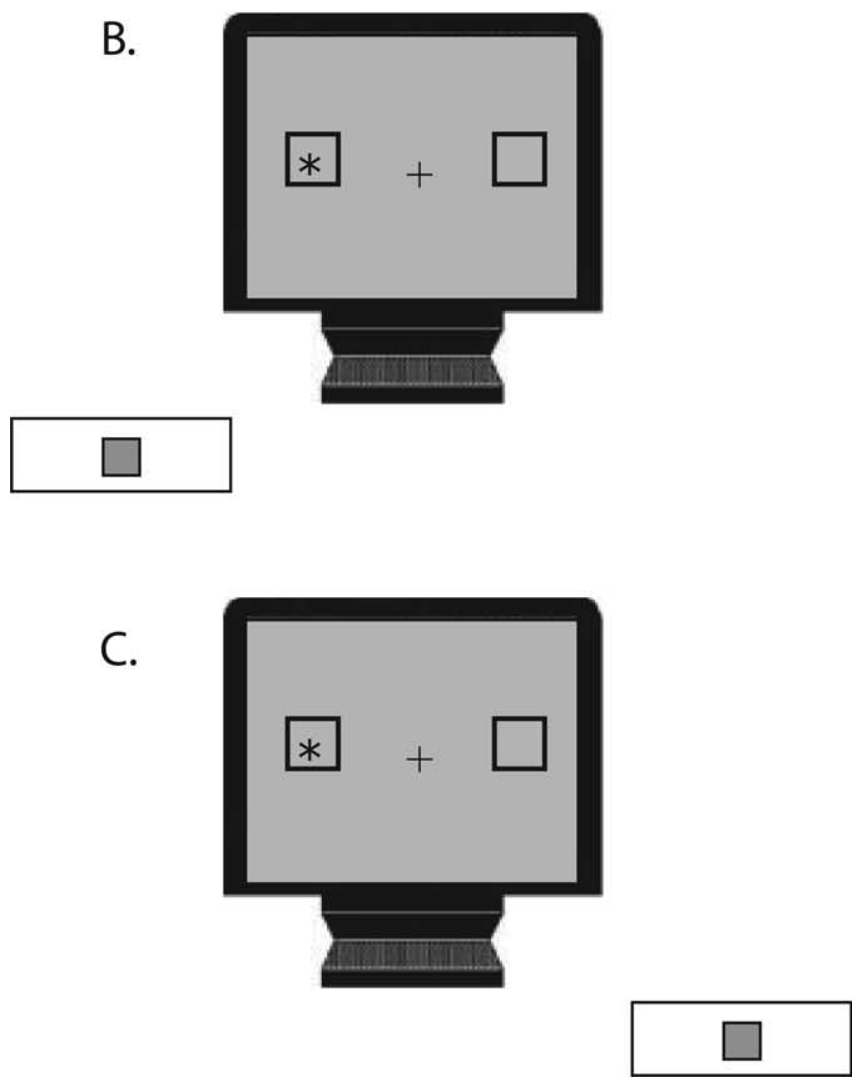

Figure 1. Experimental design. $\boldsymbol{A}$, Central starting position. The keypad was placed on the desk, aligned with the central fixation cross. Under this condition, right targets require rightward movements, and left targets require leftward movements. $\boldsymbol{B}$, Left starting position. The keypad was placed to the left of the left target location, so both right and left visual field targets (left target shown) require rightward movement. C, Right starting position. The keypad was placed to the right of the right target location, so both right and left visual field targets (left target shown) require leftward movement.

tion cross at the center of the screen and two boxes (unfilled squares), each $3^{\circ}$ on a side, plotted with their centers $12^{\circ}$ to the right and to the left of the fixation cross (Fig. 1). The target stimulus was a $1.4^{\circ}$ asterisk that appeared inside one of the peripheral boxes. The target was presented at the right and left side of the screen in equal probability.

Procedure. Each trial started with the subject pressing a key with his/her right index finger. After a random interval (1500-3000 ms), a target (asterisk) was presented for $2000 \mathrm{~ms}$ at either a left or right location. We used long target durations to minimize problems with working memory. The subject released the key as fast as possible and pointed to the target location on the screen. After touching the screen, he/she returned the 
finger to the keypad, and after a fixed interval of $500 \mathrm{~ms}$, the next trial began. Central fixation was emphasized during the trial, and eye position was monitored by an experimenter sitting in front of the patient. Three conditions were run to dissociate the position of the target and the direction of reach: central starting position (Fig. $1 \mathrm{~A}$ ), left starting position (Fig. $1 B$ ), and right starting position (Fig. 1C). Each block contains 30 trials ( 15 left and 15 right). Three blocks were run in each patient, one for each starting position. As in Mattingley et al. (1998), the central starting position combines perceptual and motor components, because left targets appear in the left visual field and require leftward movements. In contrast, the left starting position dissociates perceptual from motor neglect because left targets appear in the left visual field but require rightward movements (Fig. 1).

\section{Anatomical imaging and lesion segmentation}

Structural images were acquired on a subgroup of 29 patients (see above, Patients, Exclusion criteria) using multiple sagittal T1-weighted (magnetization-prepared rapid-acquisition gradient echo), optimized for contrast-to-noise ratio and resolution (echo time, $4 \mathrm{~ms}$; repetition time, $9 \mathrm{~ms}$; inversion time, $300 \mathrm{~ms}$; flip angle, $12^{\circ}$; 128 slices; $1 \times 1 \times 1.25$ mm voxels) (Epstein et al., 1994) and T2-weighted (fast turbo spin echo) sequences. Using in-house software, these images were realigned and averaged to increase signal-to-noise and white-gray matter contrast. Lesion boundaries were determined with the aid of an unsupervised fuzzy class means-based segmentation procedure. We automatically corrected for image intensity inhomogeneity using a variant of Styner (Styner et al., $2000)$ and the assumption that the objects imaged in the Siemens (Munich, Germany) circularly polarized head coil exhibit a parabolic threedimensional gain field (10 free parameters). Voxels were classified into one of four tissue types: air, CSF, gray matter, and white matter. A definite determination of lesion boundaries was established by a boardcertified neurologist (M.C.) using Analyze AVW4 (Mayo Foundation for Medical Education and Research, Rochester, MN). Expert judgment is required to definitively determine the lesion boundaries (e.g., to distinguish the cystic cavity from ventricle). Semiautomated segmentation significantly decreases the variability associated with manual tracing.

\section{Results}

Figure 2 shows mean reaction time (RT) to release the key (initiation of a reach) for contralesional (left) and ipsilesional (right) targets for the 52 patients and 30 controls, showing that neglect patients responded more slowly to left targets (contralesional, $-880.7 \mathrm{~ms})$ than right targets $\left(663.5 \mathrm{~ms} ; F_{(1,50)}=78.7 ; p<\right.$ $0.0001)$. Control subjects showed a very small, though significant, difference in RT for the two targets (left targets, $495.1 \mathrm{~ms}$; right targets, $\left.482.4 \mathrm{~ms} ; F_{(1,29)}=4.9 ; p<0.05\right)$. Because subjects were using their right hand, this difference may reflect the Simon effect (Simon and Rudell, 1967): faster response time to targets appearing at a spatial location compatible with the hand used for response than to targets at an opposite location (in this case, when responding with the right hand, right targets are more compatible, hence faster, than left targets). A significant visual field $\times$ group interaction revealed that patients were disproportionately slower than control subjects in the left (contralesional) visual fields $\left(F_{(1,79)}=40.1 ; p<0.0001\right)$. Note that overall, neglect patients (and controls) showed no effect of starting position $\left(F_{(2,100)}<1\right)$.

We next divided the group of patients based on whether or not they showed $\mathrm{DH}$, defined as a relative slowing in the ability to detect contralesional (left visual field) targets when reaching in a leftward direction (from a central position) compared with a rightward direction (from a left position). The first index of $\mathrm{DH}$ was the difference in the visual field bias as function of starting position: [(central position (RT left VF target - RT right VF target) $>$ left starting position (RT left VF target - right VF target)]. This index, for the most part, isolated patients with a
A.

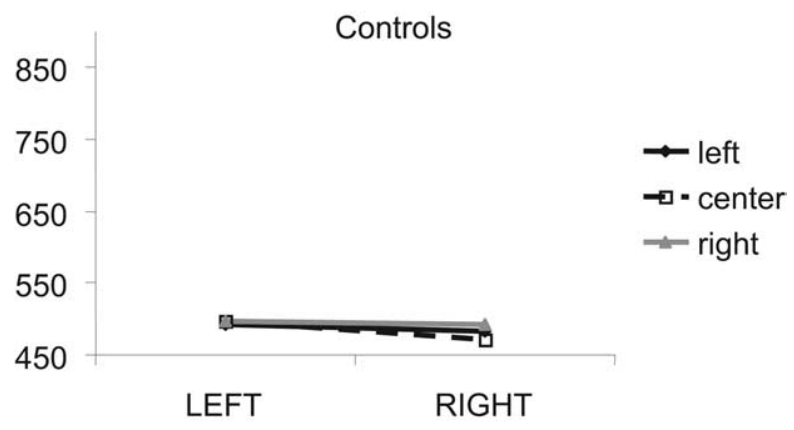

B.

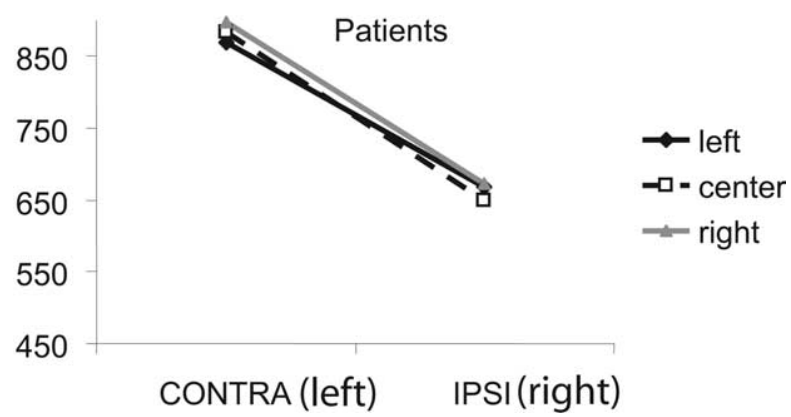

Figure 2. Behavioral results. $\boldsymbol{A}, \boldsymbol{B}$, Mean RT (in milliseconds) for initiating a motor response for left [contralesional (CONTRA) for the patients] and right [ipsilesional (IPSI) for the patients] targets, when starting at a left starting position (black solid line, filled diamond), central starting position (black dashed line, open square) and right starting position (gray solid line, filled triangle) for controls $(\boldsymbol{A})$ and patients $(\boldsymbol{B})$.

more rapid left visual field detection when starting from the left starting position (rightward direction). However, some patients showed this effect as a result of long RT to targets in the right (ipsilesional) visual field when starting from a left position. Therefore, these patients did not respond more quickly to left visual field targets when starting from the left, but rather were slower in responding to right visual field targets when starting from the left. Because the latter condition involves planning a movement across the body midline, which may be more incompatible from a motor standpoint, we devised a second index that more directly established an advantage for left visual field targets when reaching in a rightward (ipsilesional) than leftward (contralesional) direction: [ (central starting position (RT left VF target) $>$ left starting position (RT left VF target)]. Only patients who showed both effects were considered to have $\mathrm{DH}$.

Selecting an appropriate cutoff for behavioral indexes may be challenging when there is no a priori information regarding the underlying distribution. In this case, we used the distribution of scores in control subjects, normalized to a $z$-distribution, as the control population and compared each patient's score after transformation to a $z$-score value to the distribution of control $z$-scores. A patient was considered to have $\mathrm{DH}$ or a motor bias if his/her $z$-score fell below -1.64 (significance level of 0.05 ).

This procedure led to nine patients with and 43 patients without a motor bias. This procedure yielded a significant difference in the expected direction: visual field (contralateral or ipsilateral) $\times$ starting position (left, center, or right) $\times$ group (with or without motor bias) was significant $\left(F_{(2,98)}=12.73 ; p<0.0001\right)$. A subgroup of these patients also had anatomical scans. Among these patients, six had the motor bias, and 23 did not have the effect. Visual field $\times$ starting position $\times$ group was significant 
A.

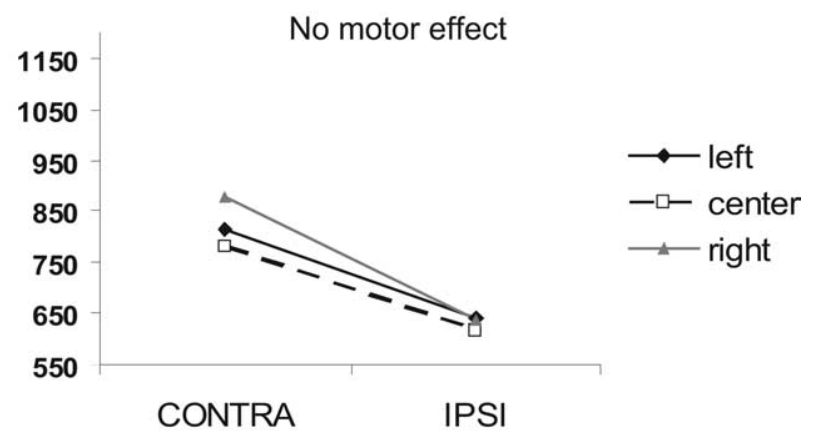

B.

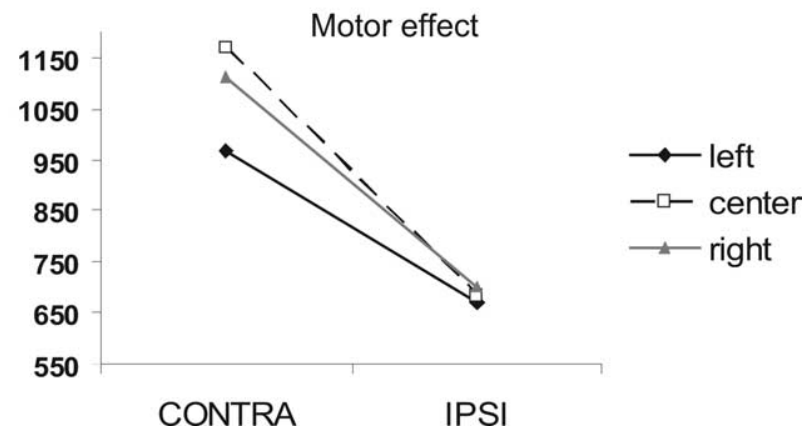

Figure 3. Behavioral results. $\boldsymbol{A}, \boldsymbol{B}$, Mean $\mathrm{RT}$ (in milliseconds) for initiating a motor response for contralesional (CONTRA; left) and ipsilesional (IPSI; right) targets, when starting at a left starting position (black solid line, filled diamond), central starting position (black dashed line, open square), and right starting position (gray solid line, filled triangle), for patients that did not show DH $(\boldsymbol{A})$ and patients that showed DH $(\boldsymbol{B})$.

also in this subgroup $\left(F_{(2,54)}=3.45 ; p<0.05\right)$. From here on, all statistics and figures presented are from the subgroup of patients with anatomy scans (Fig. 3). On average, patients with $\mathrm{DH}$ also had a larger visual field effect (visual field $\times$ group, $F_{(1,27)}=7.5$; $p<0.05$ ), which can imply more severe neglect. To further explore whether a visual field bias could explain $\mathrm{DH}$, we ran a correlation analysis between visual field deficit and DH. VF deficit was not correlated with the first or the second index of $\mathrm{DH}$ ( $r=0.17, p=0.38$ and $r=0.12, p=0.54$, respectively), suggesting that the size of the VF effect was not correlated with the size of the DH effect in the entire group. Furthermore, the anatomy of $\mathrm{DH}$ differed from the anatomy of VF bias. The lesion distribution of patients with the most severe VF bias (based on a median split) hardly overlapped with the lesion distribution of patients with DH (see supplemental Fig. 1, available at www.jneurosci.org as supplemental material). This clearly shows that the specific pattern of overlap found in ventral lateral putamen could not have been replicated if VF scores were used instead. Hence, VF deficits cannot account for the tight behavioral-anatomical correlation demonstrated for DH. Patients with $\mathrm{DH}$ also did not have larger lesions or overall more severe neglect. A comparison of lesion size showed no significant difference between patients with and without $\mathrm{DH}$ (lesion size $\times$ group, $F<1$ ). Furthermore, the two groups did not differ on many of our neuropsychological measures of extrapersonal and personal neglect. Table 1 shows that the two groups had similar scores on line cancellation, star cancellation (BIT), and symbol cancellation (Mesulam), personal neglect (Bisiach and Fluff tests), and ecological tasks of neglect (baking tray) (for similar results, see Mattingley et al., 1992). Interestingly, the two groups differed in paragraph reading and clock drawing. The difference in clock drawing can be explained by the complex motor sequences require for drawing a clock. The difference in reading text is more puzzling, and additional work is required to explain it. Note that for the reading test, the scores from only two patients drive this result, but there was no good reason to take them out of the analysis.

The anatomy of DH was investigated by plotting the lesion distribution in patients with and without a motor bias (Fig. $4 A, B)$. In patients with a motor bias, the maximum lesion overlap was centered in the putamen and claustrum (100\% of the patients), followed by the white matter underlying the frontal lobe in correspondence to precentral gyrus, inferior frontal gyrus, frontal operculum, and anterior insula ( $80 \%$ of the patients). Note that patients who showed a motor bias had a very similar lesion distribution, with values of overlap ranging from 65 to $100 \%$ in the above structures. In contrast, patients with no motor bias had much larger variability in the distribution of lesions. To identify the brain structures that are commonly damaged in patients with motor bias but are intact in patients without motor bias, we subtracted the lesions of the latter group from the lesions of the first group (Fig. 4C). We found that the ventral lateral part of the putamen was damaged $100 \%$ more often in the $\mathrm{DH}$ group than in the rest of the patients; that is, all of the patients who showed motor bias had a ventral lateral putamen lesion, whereas no patient who did not have a motor bias was damaged in that region (for regions with $>70 \%$ damage, see Fig. $4 D$ ).

To compute the probability that all six patients with $\mathrm{DH}$ had lesions overlapping the same portion of ventral lateral putamen but none of the remaining 23 neglect patients did, we used the following formula: $p=(n-d h) ! d h ! / n !$, where $n$ is the number of neglect patients, and $d h$ is the number of neglect patients with $\mathrm{DH}$. The above ratio has as a numerator the product of the number of permutations of $d h$ patients (with a lesion in ventral lateral putamen) and $n-d h$ patients (without a lesion in ventral lateral putamen) and as a denominator the number of permutations of $n$ patients. This ratio is highly significant, with a value of $3 \times 10^{-6}$ for $n=29$ and $d h=6$.

Control analyses showed that these results do not depend on the presence or distribution of lacunes in the white matter (see supplemental Table 1, available at www.jneurosci.org as supplemental material).

\section{Discussion}

Our experiment was designed to investigate the anatomical correlate of $\mathrm{DH}$ in patients with neglect. The comparison of neglect patients with and without motor bias clearly demonstrates that directional motor bias in neglect is associated with damage of the ventral lateral putamen and claustrum and to a lesser degree of the white matter underlying the frontal lobe in front of the central sulcus. Our results are consistent and extend the existing literature. The right putamen and the caudate are common sites of damage in neglect patients (Karnath et al., 2002), but their contribution to the syndrome has not been well characterized.

There are several lines of evidence that decreased levels of dopamine in the nigrostriatal pathway (from the substantia nigra to the putamen and the caudate) may contribute to neglect. For instance, unilateral reduction of dopamine in the rat caudate impairs initiation of contralateral responses but not detection of contralateral stimuli (Carli et al., 1985). Partial reduction of dopamine content in the putamen and caudate nucleus in monkeys results in slowness in initiating movements with the ipsilesional arm toward contralesional stimuli (Apicella et al., 1991). Monkeys with unilateral injections of 6-OHDA in the substantia nigra 
Table 1. Neuropsychological tests of patients with and without directional hypokinesia

\begin{tabular}{|c|c|c|c|c|}
\hline & Directional hypokinesia (mean \pm SD) & No directional hypokinesia (mean \pm SD) & Fscore & $p$ value \\
\hline Sex & $3 \mathrm{M}, 3 \mathrm{~F}$ & $15 \mathrm{M}, 8 \mathrm{~F}$ & & \\
\hline Age & $61.17 \pm 14.4$ & $59.52 \pm 14$ & $<1$ & ns \\
\hline Short Blessed test & $3.5 \pm 4$ & $3.7 \pm 4.5$ & $<1$ & ns \\
\hline Mesulam (contra - ipsi) & $6.3 \pm 8.8$ & $6.94 \pm 6.6$ & $<1$ & ns \\
\hline Mesulam (total misses) & $23.7 \pm 18.2$ & $18.9 \pm 16.2$ & $<1$ & ns \\
\hline Star cancellation (contra - ipsi) & $8.4 \pm 8.1$ & $5.13 \pm 8.4$ & $<1$ & ns \\
\hline Star cancellation (total misses) & $17.2 \pm 17.6$ & $13.3 \pm 15$ & $<1$ & ns \\
\hline Line cancellation (contra - ipsi) & $0.4 \pm 1.5$ & $0.6 \pm 3$ & $<1$ & ns \\
\hline Line cancellation (total misses) & $0.8 \pm 1.3$ & $0.86 \pm 2.9$ & $<1$ & ns \\
\hline Baking tray (ipsi - contra) & $5.8 \pm 6$ & $7.1 \pm 7.4$ & $<1$ & ns \\
\hline Bisiach personal neglect & $0.5 \pm 0.58$ & $0.3 \pm 0.47$ & $<1$ & ns \\
\hline Fluff test & $1.7 \pm 2.1$ & $0.65 \pm 1$ & 1.94 & 0.18 \\
\hline Clock drawing & $5.4 \pm 2.3$ & $1.4 \pm 1.3$ & 27.26 & 0.0001 \\
\hline BIT reading (contra - ipsi) & $21.8 \pm 26.2$ & $0.6 \pm 6.8$ & 12.5 & 0.002 \\
\hline BIT reading (total misses) & $37.5 \pm 43.3$ & $6.8 \pm 10.3$ & 9.97 & 0.004 \\
\hline
\end{tabular}

Note that all tests were inverted to one direction, so that a higher score indicates more severe neglect. F, Female; M, male; ns, not significant; contra, contralesional; ipsi, ipsilesional.

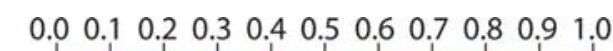

A.

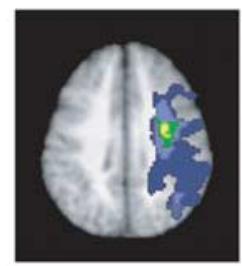

B.

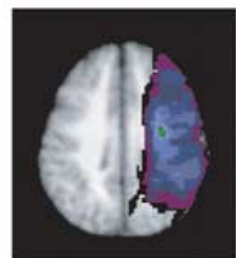

$Z=36$

c.

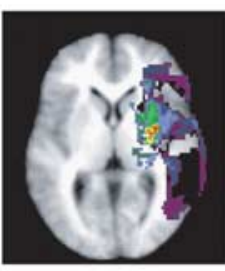

D.

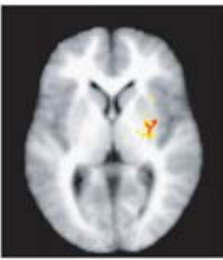

$Z=4$
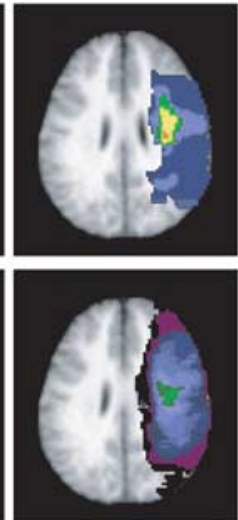

$Z=28$
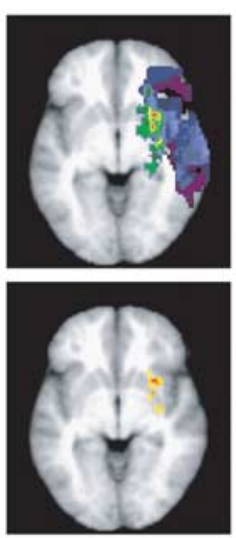

$Z=-4$
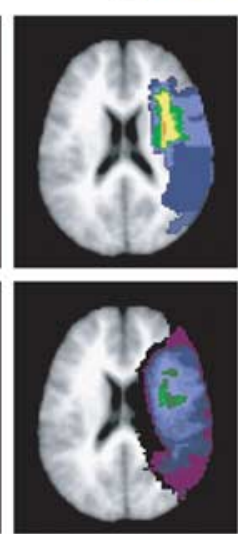

$Z=20$
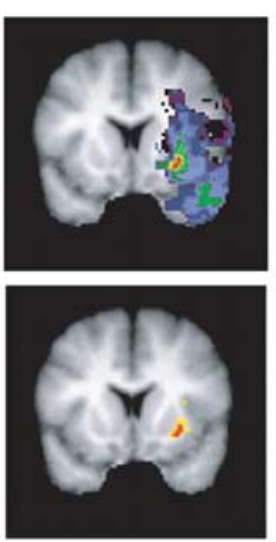

$$
\mathrm{Y}=9
$$

0.7
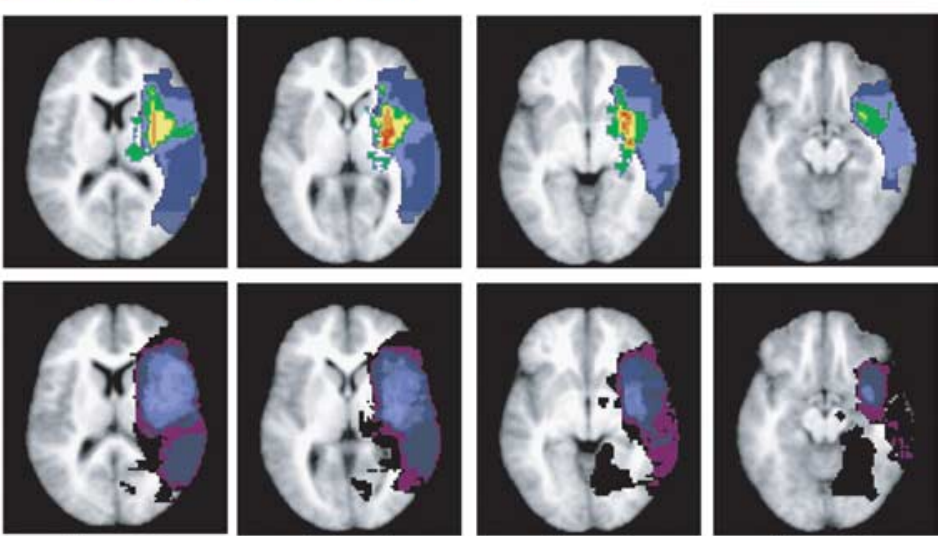

$Z=12$

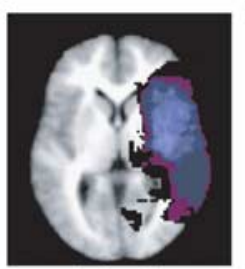

$Z=4$
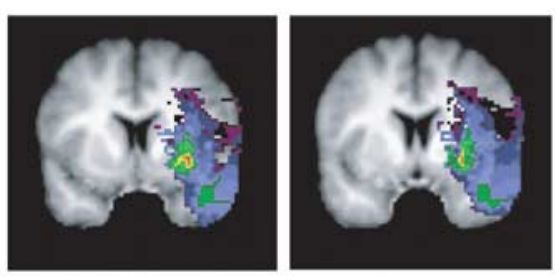

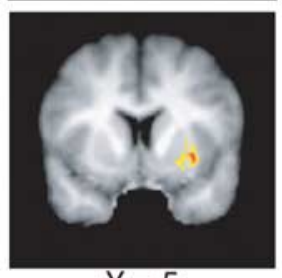

$Y=5$

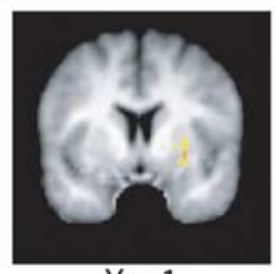

$\mathrm{Y}=1$

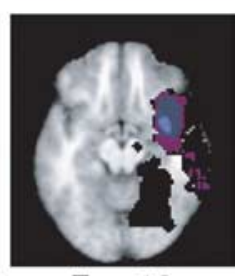

$Z=-12$

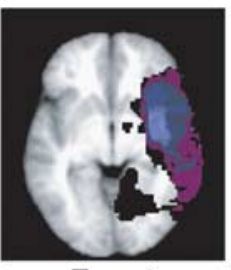

$Z=-4$
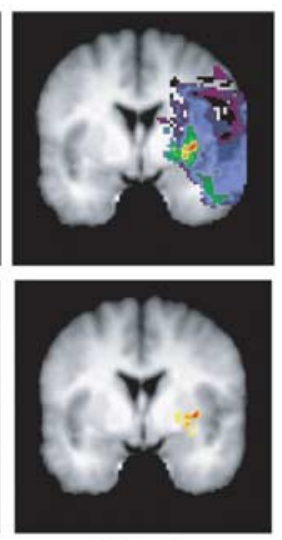

$Y=-3$

1.0

Figure 4. Anatomical analysis. $\boldsymbol{A}, \boldsymbol{B}$, Lesion overlap in the group of patients with $\mathrm{DH}(\boldsymbol{A} ; n=6)$ and without $\mathrm{DH}(\boldsymbol{B} ; n=23)$. The color scale indicates the percentage of patients with damage to a specific voxel, with red being $100 \%$ of the patients. $\boldsymbol{C}$, A difference image of the two groups (with DH minus without DH). The color scale is the same as in $\boldsymbol{A}$ and $\boldsymbol{B}$. The color scale indicates the percentage of patients with damage to a specific voxel in the group with $\mathrm{DH}$ after taking out patients without $\mathrm{DH}$, with red being $100 \%$ of the patients. D, As in C, but showing only voxels that were damaged in $>70 \%$ of the patients.

and $\sim 90 \%$ loss of cells in the caudate, putamen, nucleus accumbens, and substantia nigra showed more body rotations toward the lesion side and persistent head deviation toward the ipsilesional side. In addition, these animals had problems reaching into contralesional space, using both the good and the impaired arm (Milton et al., 2004).

Furthermore, modulation of dopamine transmission by drugs modulates neglect. Bromocriptine, a dopamine agonist, can sig- 
nificantly improve neglect (Fleet et al., 1987). Similar results have been reported with apomorphine, a different dopamine agonist (Geminiani et al., 1998). In the latter study, in three of four treated patients, the improvement was more pronounced in the motor task (pointing) than in the perceptual task (counting), suggesting that decreased dopamine level may mediate the motor component of neglect. In another single case study, treatment with bromocriptine of a patient with motor intentional neglect and a putamen lesion increased the motor bias toward the ipsilesional field (Barrett et al., 1999). The authors suggested that the right side putamen lesion might have prevented the positive effect of bromocriptine on the right hemisphere, while at the same time stimulating the intact left putamen, which in turn caused increased motor bias (for a group study with similar results, see also Grujic et al., 1998). Overall, these studies strongly support the involvement of the dopamine system in neglect and in particular the involvement of the putamen in mediating the motor bias in neglect.

How do our results compare with previous localization of DH? Several years ago, Mattingley et al. (1992) found that a group with anterior/subcortical lesions showed longer movement times for executing sequences in a leftward than rightward direction. This result is consistent with our behavioral results, but the anatomical mapping procedures did not allow a more precise localization of the site of damage. Many previous studies pointed to the frontal lobe as the most likely site of damage for motor neglect (Daffner et al., 1990; Tegner and Levander, 1991; Bottini et al., 1992), but later it was argued that most of the tests designed to measure DH used a noncompatible condition to dissociate motor from perceptual components in neglect. Hence, the frontal localization may not be a function of the directional motor component, but of the well known difficulty of frontal patients with noncompatible conditions (Husain et al., 2000). In our study, the white matter underlying the location of putative dorsal and ventral premotor cortices (i.e., precentral and inferior frontal gyri) was consistently damaged in patients with motor bias, supporting a deficit of motor planning in $\mathrm{DH}$. In contrast to this more anterior (caudate, putamen, and frontal lobe) localization, Mattingley et al. (1998) found that patients with inferior parietal lesions, but not patients with inferior frontal lesions, showed $\mathrm{DH}$.

We suggest that a critical region for $\mathrm{DH}$ is the ventral lateral putamen, but we do not claim that this region alone is responsible for DH. Rather, the putamen may be part of a network that is important for motor execution or exploratory behavior of the left side of space. Damage to one node of the network produces widespread functional impairment in other nodes of the network, which may in turn result in similar behavioral deficits. In support of this view, blood flow studies indicate that subcortical lesions cause cortical hypoperfusion (Perani et al., 1987; Vallar et al., 1988; Hillis et al., 2002, 2005). In particular, basal ganglia lesions that cause neglect are associated with abnormal perfusion of the superior temporal gyrus, inferior parietal lobule, and inferior frontal gyrus (Karnath et al., 2005). These findings would reconcile ours with the localization of DH to the inferior parietal lobule by Mattingley et al. (1998). It is possible that more than one site is involved in DH and different groups of patients will show different lesions causing the same behavioral deficit, because these lesions are all part of the same network. Our data do not allow us to determine whether $\mathrm{DH}$ is directly attributable to the putaminal lesion or mediated by cortical dysfunction (for instance, in the inferior parietal lobule) induced by the putaminal lesion. Distinguishing between these mechanisms would require measuring neural or neurally driven metabolic activity in the inferior pari- etal lobule of subjects with and without putaminal lesions and with or without $\mathrm{DH}$.

These results have obvious implications for designing novel rehabilitation strategies after neurological injuries. It may be possible to differentiate neglect in subgroups based on their behavioral profile or functional neuroimaging patterns of dysfunction/ recovery; each subtype may differently impact long-term outcome and be amenable to specialized rehabilitation interventions. For example, patients with $\mathrm{DH}$ with putaminal lesions may benefit from agents affecting dopaminergic transmission, whereas patients with other forms of neglect would not.

\section{References}

Apicella P, Legallet E, Nieoullon A, Trouche E (1991) Neglect of contralateral visual stimuli in monkeys with unilateral striatal dopamine depletion. Behav Brain Res 46:187-195.

Barrett AM, Crucian GP, Schwartz RL, Heilman KM (1999) Adverse effect of dopamine agonist therapy in a patient with motor-intentional neglect. Arch Phys Med Rehabil 80:600-603.

Bisiach E, Vallar G, Perani D, Berti A (1986) Unawareness of disease following lesions of the right hemisphere: anosognosia for hemiplegia and anosognosia for hemianopia. Neuropsychologia 24:471-482.

Bisiach E, Geminiani G, Berti A, Rusconi ML (1990) Perceptual and premotor factors of unilateral neglect. Neurology 40:1278-1281.

Bottini G, Sterzi R, Vallar G (1992) Directional hypokinesia in spatial hemineglect: a case study. J Neurol Neurosurg Psychiatry 55:562-565.

Buxbaum LJ, Ferraro MK, Veramonti T, Farne A, Whyte J, Ladavas E, Frassinetti F, Coslett HB (2004) Hemispatial neglect: subtypes, neuroanatomy, and disability. Neurology 62:749-756.

Carli M, Evenden JL, Robbins TW (1985) Depletion of unilateral striatal dopamine impairs initiation of contralateral actions and not sensory attention. Nature 313:679-682.

Chiba Y, Yamaguchi A, Eto F (2005) A simple method to dissociate sensoryattentional and motor-intentional biases in unilateral visual neglect. Brain Cogn 58:269-273.

Cocchini G, Beschin N, Jehkonen M (2001) The Fluff test: a simple task to assess body representation neglect. Neuropsychol Rehabil 11:17-31.

Coslett HB, Bowers D, Fitzpatrick E, Haws B, Heilman KM (1990) Directional hypokinesia and hemispatial inattention in neglect. Brain 113:475-486.

Daffner KR, Ahern GL, Weintraub S, Mesulam M-M (1990) Dissociated neglect behavior following sequential strokes in the right hemisphere. Ann Neurol 28:97-100.

Epstein FH, Mugler JPI, Brookeman JR (1994) Optimization of parameter values for complex pulse sequences by simulated annealing: application to 3D MP-RAGE imaging of the brain. Magn Reson Med 31:164-177.

Fleet W, Valenstein E, Watson R, Heilman K (1987) Dopamine agonist therapy for neglect in humans. Neurology 37:1765-1770.

Geminiani G, Bottini G, Sterzi R (1998) Dopaminergic stimulation in unilateral neglect. J Neurol Neurosurg Psychiatry 65:344-347.

Grujic Z, Mapstone M, Gitelman DR, Johnson N, Weintraub S, Hays A, Kwasnica C, Harvey R, Mesulam MM (1998) Dopamine agonists reorient visual exploration away from the neglected hemispace. Neurology 51:1395-1398.

Heilman KM, Watson RT, Valenstein E, Damasio AR (1983) Localization of lesions in neglect. In: Localization in neuropsychology (Kertesz A, ed), pp 471-492. New York: Academic.

Heilman KM, Bowers D, Coslett HB, Whelan H, Watson RT (1985) Directional hypokinesia: prolonged reaction times for leftward movements in patients with right hemisphere lesions and neglect. Neurology 35:855-859.

Heilman KM, Bowers D, Valenstein E, Watson RT (1993) Disorders of visual attention. Baillieres Clin Neurol 2:389-413.

Hillis AE, Wityk RJ, Barker PB, Beauchamp NJ, Gailloud P, Murphy K, Cooper O, Metter EJ (2002) Subcortical aphasia and neglect in acute stroke: the role of cortical hypoperfusion. Brain 125:1094-1104.

Hillis AE, Newhart M, Heidler J, Barker PB, Herskovits EH, Degaonkar M (2005) Anatomy of spatial attention: insights from perfusion imaging and hemispatial neglect in acute stroke. J Neurosci 25:3161-3167.

Husain M, Kennard C (1996) Visual neglect associated with frontal lobe infarction. J Neurol 243:652-657. 
Husain M, Mattingley JB, Rorden C, Kennard C, Driver J (2000) Distinguishing sensory and motor biases in parietal and frontal neglect. Brain 123:1643-1659.

Ishiai S, Sugishita M, Watabiki S, Nakayama T, Kotera M, Gono S (1994) Improvement of left unilateral spatial neglect in a line extension task. Neurology 44:294-298.

Karnath HO, Ferber S, Himmelbach M (2001) Spatial awareness is a function of the temporal not the posterior parietal lobe. Nature 411:950-953.

Karnath HO, Himmelbach M, Rorden C (2002) The subcortical anatomy of human spatial neglect: putamen, caudate nucleus and pulvinar. Brain 125:350-360.

Karnath HO, Fruhmann Berger M, Kuker W, Rorden C (2004) The anatomy of spatial neglect based on voxelwise statistical analysis: a study of 140 patients. Cereb Cortex 14:1164-1172.

Karnath HO, Zopf R, Johannsen L, Fruhmann Berger M, Nèagele T, Klose U (2005) Normalized perfusion MRI to identify common areas of dysfunction: patients with basal ganglia neglect. Brain 128:2462-2469.

Katzman R, Brown T, Fuld P, Peck A, Schechter R, Schimmel H (1983) Validation of a short orientation-memory-concentration test of cognitive impairment. Am J Psychiatry 140:734-739.

Mattingley JB, Bradshaw JL, Phillips JG (1992) Impairments of movement initiation and execution in unilateral neglect. Directional hypokinesia and bradykinesia. Brain 115:1849-1874.

Mattingley JB, Husain M, Rorden C, Kennard C, Driver J (1998) Motor role of human inferior parietal lobe revealed in unilateral neglect patients. Nature 392:179-182.

Mesulam M (1985) Mental state assessment of young and elderly adults in behavioral neurology. In: Principles of behavioral neurology (Mesulam M, ed). Philadelphia: F. A. Davis.

Mijoviâc D (1991) Mechanisms of visual spatial neglect. Absence of directional hypokinesia in spatial exploration. Brain 114:1575-1593.
Milton AL, Marshall JW, Cummings RM, Baker HF, Ridley RM (2004) Dissociation of hemi-spatial and hemi-motor impairments in a unilateral primate model of Parkinson's disease. Behav Brain Res 150:55-63.

Mort DJ, Malhotra P, Mannan SK, Rorden C, Pambakian A, Kennard C, Husain M (2003) The anatomy of visual neglect. Brain 126:1986-1997.

Pedersen PM, Jorgensen HS, Nakayama H, Raaschou HO, Olsen TS (1997) Hemineglect in acute stroke-incidence and prognostic implications. The Copenhagen stroke study. Am J Phys Med Rehabil 76:122-127.

Perani D, Vallar G, Cappa S, Messa C, Fazio F (1987) Aphasia and neglect after subcortical stroke: a clinical/cerebral perfusion correlation study. Brain 110:1211-1229.

Simon JR, Rudell AP (1967) Auditory S-R compatibility: the effect of an irrelevant cue on information processing. J Appl Psychol 51:300-304.

Styner M, Brechbuhler C, Szekely G, Gerig G (2000) Parametric estimate of intensity inhomogeneities applied to MRI. IEEE Trans Med Imaging 19:153-165.

Tegner R, Levander M (1991) Through a looking glass. A new technique to demonstrate directional hypokinesia in unilateral neglect. Brain 114:1943-1951.

Tham K, Tegner R (1996) The baking tray task: a test of spatial neglect. Neuropsyc Rehab 6:19-25.

Vallar G (2001) Extrapersonal visual unilateral spatial neglect and its neuroanatomy. NeuroImage 14:S52-S58.

Vallar G, Perani D (1986) The anatomy of unilateral neglect after righthemisphere stroke lesions. A clinical/CT-scan correlation study in man. Neuropsychologia 24:609-622.

Vallar G, Perani D, Cappa SF, Messa C, Lenzi GL, Fazio F (1988) Recovery from aphasia and neglect after subcortical stroke: neuropsychological and cerebral perfusion study. J Neurol Neurosurg Psychiatry 51:1269-1276.

Wilson B, Cockburn J, Halligan P (1987) Development of a behavioral test of visuospatial neglect. Arch Phys Med Rehabil 68:98-102. 